Wheatfields hospice. We conducted 4 focus groups with six service users and 17 professionals involved in palliative care. We analysed these using thematic analysis with two independent coders.

Findings We found several major themes. Service users identified access to their GP as a major barrier. Service users and healthcare professionals also identified discrimination and lack of trust in professionals. Healthcare professionals identified a lack of experience and education in caring for this group, but stated they were keen to develop this. Service users also identified education around what palliative care is as a major barrier to accessing services. Despite these themes both professionals and service users described their real life experience as positive.

Conclusion Although the experience of both service users and professionals in the context of palliative care in this community has been positive we have identified barriers to developing these services further. Flexible services are required to ensure holistic, and culturally sensitive care. This requires collaboration between services, with access to GPs being identified as a crucial area to start.

\section{RESPECT, FIND OUT WHAT IT MEANS TO ME?}

Natalie Wright, Sinead Henderson. Ashford and St Peter's NHS Trust

10.1136/spcare-2020-PCC.183

Introduction ReSPECT is a national initiative to encourage shared decision making around treatment decision and advance care planning. We undertook a trust wide launch to replace all DNAR forms in November 2018. The aim of this project was to evaluate staff understanding of the respect process and confidence in having significant conversations, as part of a wider trust evaluation.

Methods In dying matters week the Specialist Palliative care team (SPCT) designed a staff survey to rate confidence and understanding of the Respect process, staff were asked a number of questions including; completion of online ReSPECT training app, understanding of the respect process and their role and confidence in participating in conversations with patients about their wishes around future treatment and care. SPCT visited all areas of the hospital and asked staff to complete the questionnaire.

Results 132 health care professionals replied. 44\% doctors, $37 \%$ Nurses, 19\% Allied health professionals. 93\% were aware that ReSPECT has been introduced to the trust, only $17 \%$ had completed the online training. $83 \%$ of respondents felt strongly confident or confident to discuss with patients their wishes about their care. Only 59\% felt strongly confident or confident to discuss resuscitation status and end of life care. However, $100 \%$ senior nurses and doctors felt confident or strongly confident in discussing resuscitation status and end of life care.

Conclusions It is reassuring that our senior staff feel confident in engaging patients in such significant conversations. We are exploring making the ReSPECT learning app completion mandatory to further support all staff and recognise the need to empower senior nursing staff to participate further in the ReSPECT process in our trust. We will use this information to promote significant conversations as a process rather than a one off and improve form completion.

\section{Supportive care | posters $164-179$}

\section{COMPASSIONATE COMMUNITIES': A QUALITATIVE STUDY EXPLORING THE VIEWS OF GENERAL PRACTITIONERS}

Elizabeth Abbey, Claire Craig, Catriona Mayland. University of Sheffield, Sheffield Hallam University

\subsection{6/spcare-2020-PCC. 184}

Introduction General Practitioners (GPs) face challenges when providing palliative care, including an ageing, multimorbid population, falling GP numbers and increasing demand. A 'public health palliative care' (PHPC) approach is gaining momentum, and is defined as 'working with communities to improve people's experience of death, dying and Bereavement'. 'Compassionate communities' is one example, supporting patients by linking professional health carers and community networks. Primary care is central to the approach, which has been incorporated into General Practice Daffodil Standards, however there is little evidence of GP perspectives of these approaches. Our aim, therefore, was to explore GP perceptions of PHPC and 'compassionate communities'.

Methods Using snowball sampling, GPs were recruited through university teaching and research networks. Additionally, purposive sampling ensured wide representation of gender, level of experience and practice populations. Following informed consent, semi-structured, digitally audio-recorded interviews were conducted. Interviews were transcribed verbatim, and thematic analysis was undertaken. Interviews continued until data saturation was reached.

Results Nine GPs were interviewed. Most were unfamiliar with 'compassionate communities', but recognised examples within their practice. Three themes identified perceived benefits of the model: 'increasing awareness of community services'; 'combatting taboo'; and 'embracing health outside of healthcare'. Three themes identified perceived barriers: 'limited time and funding'; 'patient safety concerns'; and 'integration with current care'. Some GPs perceived the PHPC approach as a distinct social issue outside of 'proper' palliative care and General Practice. Others envisaged a GP 'signposter' role, supporting a community-led system.

Conclusion GPs recognised the importance of the wider community in caring for palliative care patients, but had differing views regarding the application of 'compassionate communities' and their position within this. Understanding more about the model's practical implementation and exploring potential service-users' views would help establish the feasibility of 'compassionate communities' in practice.

\section{EXISTENTIAL SUFFERING IN THE DAY-TO-DAY LIVES OF THOSE LIVING WITH PALLAITIVE CARE NEEDS ARISING FROM CHRONIC OBSTRUCTIVE PULMONARY DISEASE}

Louise E Bolton, Jane Seymour, Clare Gardiner. University of Sheffield

\subsection{6/spcare-2020-PCC.185}

COPD is predicted to become the 3rd biggest cause of death worldwide by 2030 , placing palliative care provision a high 
priority. COPD palliative care provision currently focuses upon physical symptom management with less attention paid to the holistic ethos of palliation. A integrative review of existing literature across twelve databases was undertaken to explore the presence of existential suffering for those living with COPD and associated palliative care needs. 35 papers were included within the review of both qualitative and quantitative underpinnings. Thematic analysis was completed, identifying key themes explaining the impact of existential suffering:

1. 'Liminality' Patients are unsure of who they currently are and who they will become throughout disease progression.

2. 'Lamented Life' Feelings of life ceasing to exist, fuelled by grief of former life prior to illness.

3. 'Loss of Personal Liberty' - Frustration that they couldn't do activities/hobbies they once could.

4. 'Existential Isolation' Becoming isolated throughout disease progression from social and personal/family relationships. Feelings of being misunderstood had a significant impact upon low feelings of self-worth.

5. 'Life Meaning' Some patients were able to identify some life meaning and purpose, through adapting hobbies and interests to within their physical capabilities.

This integrative review has given good insight into how existential suffering manifests within this patient population. Furthermore, it has identified the need for further research to explore how life meaning and purpose is found within their daily lives, alongside the need to adapt current palliative care policy to encompass addressing existential suffering.

\section{A RENAL PALLIATIVE INTEGRATED APPROACH - IMPROVING CARE FOR HAEMODIALYSIS PATIENTS?}

Louise C Gilhooley, Jenny Bennet, Alisha Morgan. Severn Hospice, Shrewsbury and Telford NHS Trust

\subsection{6/spcare-2020-PCC.186}

Background Patients with end stage renal failure on dialysis have a high symptom burden (1) and survival on dialysis can be comparable to some cancers. (2) Increasingly it is recognised that patients with advancing age and comorbidities may benefit from a conservative approach rather than dialysis.(3) Patients with non-malignant disease often receive palliative services late or not at all.(4)

Aims Development of a monthly Renal Palliative MDT meeting alongside a Supportive Care Register (SCR) with the aim to improve renal palliative integration, education and support for patients with End Stage Renal Failure (ESRF), particularly focusing on haemodialysis (HD).

Results Data from 2018/19 collected and analysed. All deaths in patients on haemodialysis were analysed. In 2019 increasing amounts of haemodialysis patients who died had been identified and placed on SCR (48\% versus 70\%). Numbers of deaths in those not on SCR or discussed at MDT reducing. $69 \%$ of patients identified on SCR died in a community setting in 2018, this has increased in 2019 to $75 \%$ dying in a community setting.

Conclusion A SCR and monthly MDT improved access by identifying patients earlier. Numbers of deaths in those not on SCR or discussed at MDT reducing each year, suggesting we are missing less of the patients who are likely to die. Some HD deaths will be in chronic patients who we could not predict would die suddenly. The referrals have been appropriate and controlled in number. The majority of patients on SCR and discussed died out of hospital in a community setting. A greater understanding of the population we support and mutual education has resulted in closer working relationships and increased referrals. Further development of a joint Nephrology/Palliative clinic is planned. A focus group to identify patient and carer needs and experiences is planned.

\section{THE SURPRISE QUESTION IN SEVERE COPD EXACERBATIONS: CORRELATION WITH DNACPR DECISIONS AND ADVANCE CARE PLANNING}

Sarah M Gillespie, Nicholas D Lane, Katherine E Frew, Stephen C Bourke. Respiratory Research Northumbria Healthcare NHS Foundation Trust, Institute of Cellular Medicine Newcastle University, Department of Palliative Medicine Northumbria Healthcare NHS Foundation Trust, Institute of Health and Society Newcastle University

\subsection{6/spcare-2020-PCC.187}

Background Patients with COPD have a higher symptom burden than those with lung cancer. One year mortality following admission with an exacerbation of COPD (ECOPD) is 23.2\%. The 'Surprise Question' is an established screening tool designed to select patients likely to have a poor prognosis and palliative care needs. We examined whether there was a correlation between a 'No' answer to this question and evidence of prognostic or advance care planning discussions.

Methods As part of the MoSHCOPD study (clinicaltrials.gov NCT03657121) unique, consecutive patients surviving hospital admission for ECOPD were recruited. During admission, the responsible consultant was asked 'Would you be surprised if this patient died within the next year?'. On discharge, we recorded whether the patient had a Do Not Attempt Cardiopulmonary Resuscitation (DNACPR) decision in place (prognostic discussions not resulting in a DNACPR decision were not captured) and whether there was evidence of further advance care planning or specialist palliative care (SPC) input.

Results Of 314 patients recruited to date, a 'No' answer to the Surprise Question was given in 151 (48.9\%). Of these, 87 (57.6\%) had a DNACPR decision recorded on discharge and 15 (9.9\%) had evidence of either advance care planning or SPC involvement. In patients with both a 'No' answer and an active DNACPR, 15 (17.2\%) had evidence of either advance care planning or SPC involvement.

Conclusion Despite clinicians having a pessimistic expectation of one year survival in this patient group, rates of advance care planning and SPC involvement are disappointingly low, in keeping with previous research in this area. Clinicians' lack of confidence in estimating prognosis following ECOPD is likely to contribute to these findings and needs to be urgently addressed. The MoSHCOPD study aims to improve prognostication following ECOPD, thus supporting clinicians to engage in advance care planning in this underserved patient group. 